
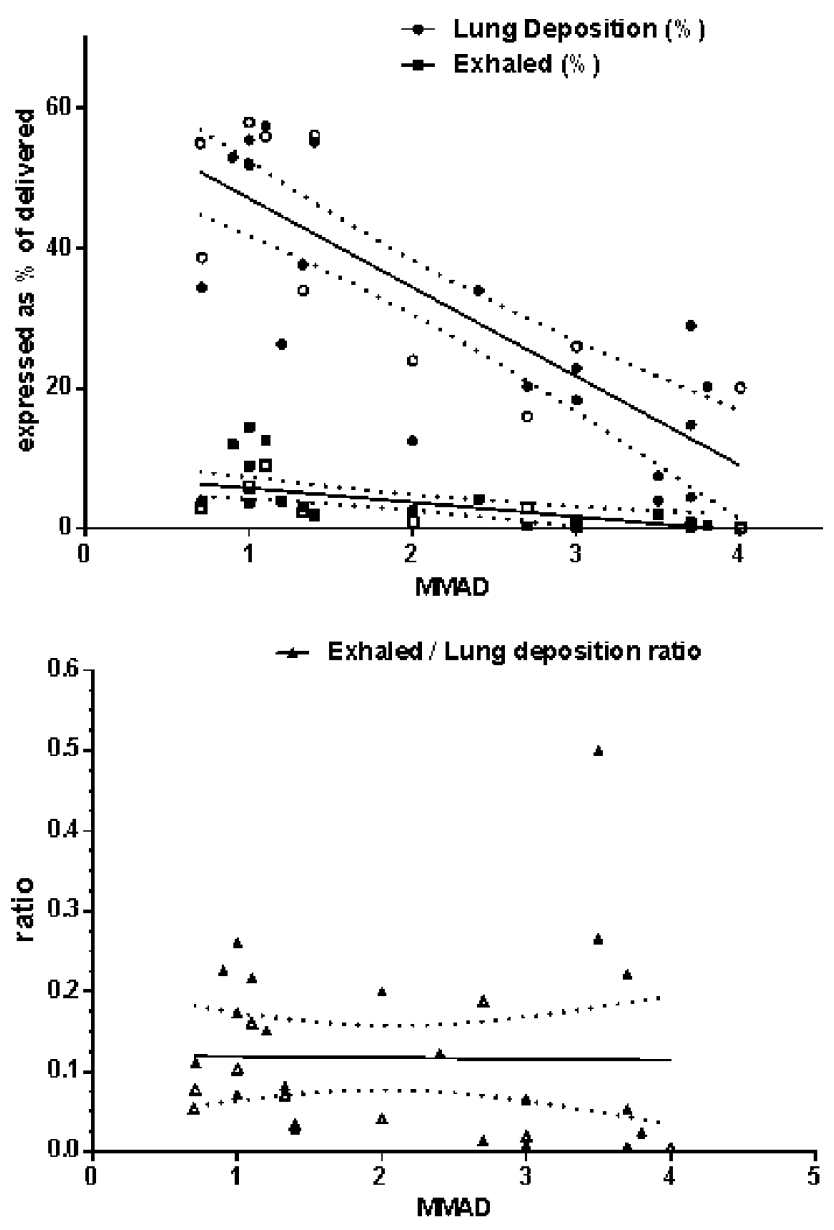

Full square, circle and triangle: healthy volunteers open square, circles and triangles: asth matic patients

Abstract P291 Figure 1 Lung deposition, exhaled fraction and ratio of exhaled vs lung deposition as a function of MMAD (regression line and the $95 \% \mathrm{Cl}$ )

Results LD increased when MMAD decreased, so that when MMAD is about $1 \mu \mathrm{m}$ the LD is more than $50 \%$ of the delivered dose and becomes markedly lower when MMAD approach 4 $\mu \mathrm{m}$. EF is low and did not change markedly. On the contrary, the ratio $\mathrm{EF} / \mathrm{LD}$ is independent of the MMAD, suggesting that the EF is proportional to the LD and not affected by MMAD. Conclusion These data demonstrate that the EF/LD ratio is independent from the MMAD providing reassurance that a smaller particle size will not be associated with a higher exhaled fraction.
Difficult symptom control and breathlessness

\begin{tabular}{l|l}
\hline M1 AN AUDIT OF ELECTRONIC OXYGEN PRESCRIBING AND \\
OXYGEN SATURATION READINGS SHOWING A HIGH \\
PREVALENCE OF RISK FACTORS FOR HYPERCAPNIA \\
AND A HIGH INCIDENCE OF IATROGENIC \\
HYPEROXAEMIA
\end{tabular}

P Whittemore, BR O'Driscoll. Salford Royal Foundation NHS Trust, Salford, UK

\subsection{6/thoraxjnl-2015-207770.428}

Background BTS audits have shown that about 14\% of UK hospital patients are on oxygen therapy at any given time but only half of these patients have a prescription or written order for oxygen use. Our 600 bed teaching hospital has electronic prescribing linked to an electronic bedside observations system (modified NEWS score). Hospital policy is to set a target oxygen saturation range for all in-patients. Patients score points if their oxygen saturation $\left(\mathrm{SpO}_{2}\right)$ falls below their target range or if $\mathrm{SpO}_{2}$ rises above their target range on oxygen therapy.

Methods We audited oxygen prescribing and $\mathrm{SpO}_{2}$ for all patients treated on medical and surgical wards during the month of December 2014. All data was contained within the electronic patient record.

Results We audited 80,391 sets of observations for 6,800 patients (2239 medical, 4561 surgical).

- $99.8 \%$ of all patients had an oxygen target range prescribed electronically

- $12.7 \%$ of all patients (18.9\% of medical patients and $9.6 \%$ of surgical patients) had risk factors for hypercapnia with a prescribed target range of $88-92 \%$ or less

- Overall $90.6 \%$ of oxygen saturation measurements were within target range (or above the target range breathing air). $59.9 \%$ of measurements on oxygen and $97.2 \%$ of observations on air were within the target range

- $3.7 \%$ of oximetry measurements were below the target range (7.9\% of those on oxygen)

- $5.8 \%$ of all oximetry measurements were above the target range due to use of oxygen $(32.2 \%$ of measurements on oxygen were above target range)

- For patients using oxygen therapy with target range 94-98\% or $88-92 \%$, the percent of measurements within range, below range or above range is shown in the Table 1

- $53.2 \%$ of observations on patients using oxygen with target range $88-92 \%$ showed $\mathrm{SpO}_{2}$ above target range

Conclusions An oxygen target range is prescribed for almost all inpatients at this hospital but hyperoxaemia (with associated risk of hypercapnia) remains prevalent amongst patients on oxygen therapy, especially those who have been identified as at increased risk of hypercapnia. This has led to a revised educational programme for nursing staff with an emphasis on keeping patients within their target range.

\section{M2 USING A TRANSPORTABLE OXYGEN CONCENTRATOR (TPOC) TO FACILITATE PROMPT AND SAFE HOSPITAL DISCHARGE}

${ }^{1} \mathrm{~F}$ Hamilton, ${ }^{2} \mathrm{G}$ Luxford, ${ }^{2} \mathrm{~J}$ Bott. ${ }^{1}$ Dolby Vivisol, Gatwick, UK; ${ }^{2} \mathrm{KSS}$ AHSN, South East England, UK

10.1136/thoraxjnl-2015-207770.429 


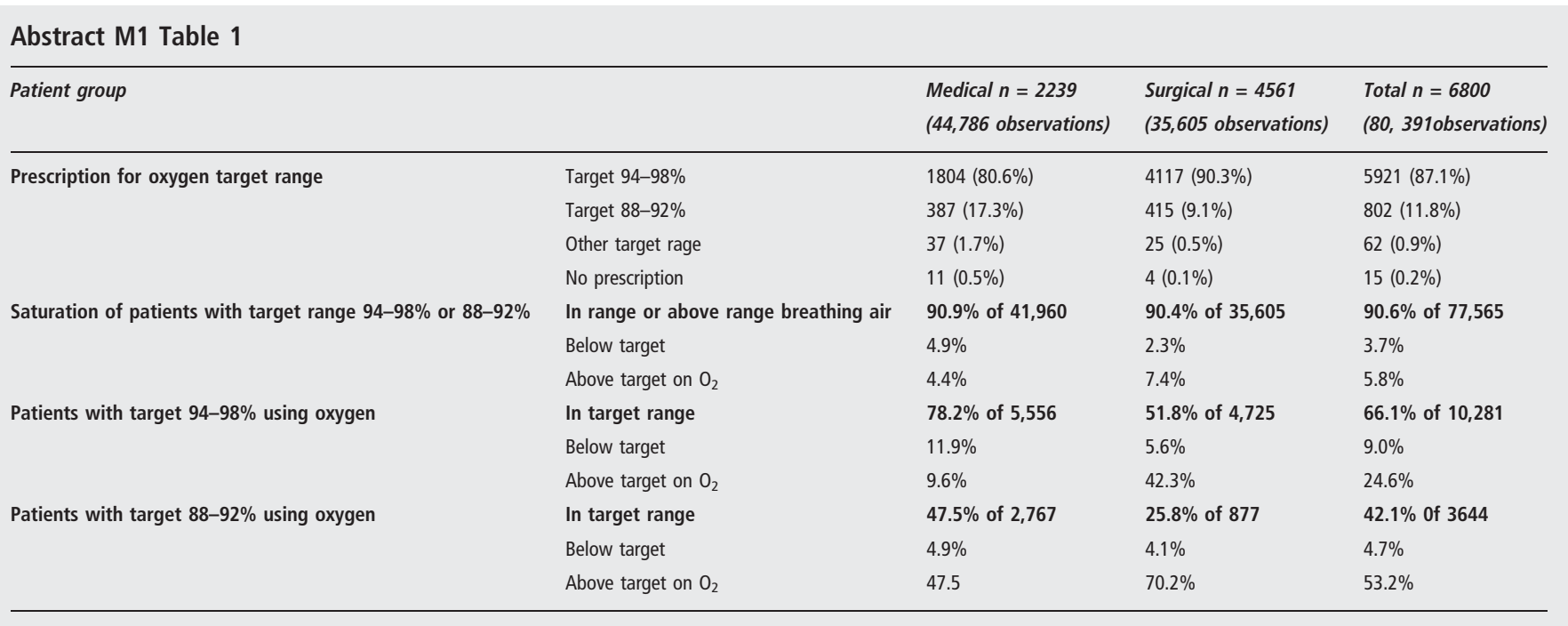

Introduction Whilst current guidelines ${ }^{1}$ state that patients must be clinically stable prior to commencing home oxygen, respiratory patients can be discharged with a supply of oxygen. Multiple errors are often found on the Home Oxygen Order Forms (HOOFs) for hospital discharges and equipment requirements are often subsequently changed, generating wasteful activity and costs. The aim was to establish whether a TPOC could be provided to specialist respiratory teams within hospitals to promote efficient and safe discharge for those patients requiring home oxygen.

Method Three hospitals with established Home Oxygen Assessment and Review Services (HOSAR) were issued with TPOCs. The Home Oxygen Supplier trained the HOSAR clinicians on use, and supplied written documentation on safety. The clinicians identified appropriate patients based on clinical assessments and issued them with a TPOC. A HOOF was then sent to the supplier with appropriate equipment for the patient's long term needs. On installation of this, the supplier removed the TPOC and another was issued to the hospital to enable an ongoing supply.

Results Of those discharged with a TPOC and a subsequent HOOF, only $5 \%(n=6)$ of patients required a modality change or HOOF update within the following month, compared to $40 \%(\mathrm{n}=33296)$ of all other HOOFs received (Figure 1$)$.

The largest group of patients issued with a TPOC on discharge were clinically coded as COPD (40\%), followed by those coded as Palliative Care (28\%).

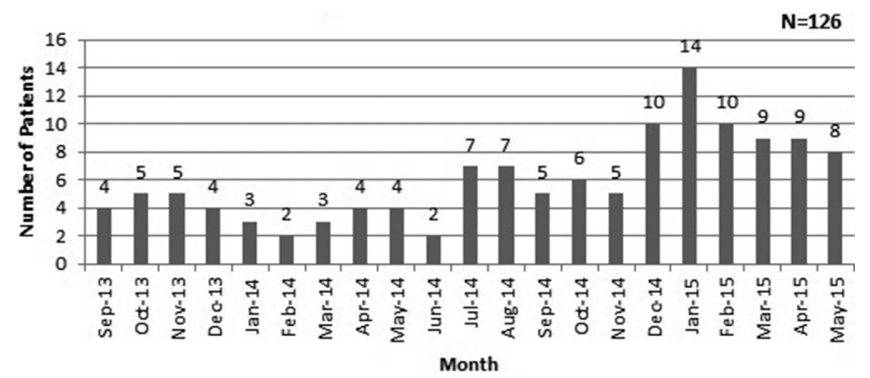

Abstract M2 Figure 1 Number of patients discharged from hospital with a TPOC

Conclusion The results demonstrate the practical uses of a TPOC for hospital discharge in clinically appropriate patients and a greater degree of control over the accuracy of ordering. A precise cost saving cannot be demonstrated as it is unclear how many bed days were saved, but it is clear that a reduction in wasteful activity by more accurate ordering would have reduced costs.

Discussion By using staff trained on equipment and accurately completing HOOFs, combined with equipment readily available, transition from hospital to home can be both clinically accurate, time efficient and cost effective.

\section{REFERENCE}

1 Hardinge $M$, Annandale J, Bourne $S$, et al. British Thoracic Society guidelines for home oxygen use in adults. Thorax 2015;70:i1-i43

\section{M3 ANXIETY AND DEPRESSION IN PATIENTS WITH BREATHING PATTERN DISORDERS OR CHRONIC RESPIRATORY DISEASE}

${ }^{1} \mathrm{SD}$ Naylor, ${ }^{2} \mathrm{~J}$ Haines, ${ }^{2} \mathrm{~A}$ Vyas, ${ }^{3} \mathrm{SJ}$ Fowler. ${ }^{1}$ University of Manchester, Manchester, UK; ${ }^{2}$ Department of Respiratory Medicine, Lancashire Teaching Hospitals NHS Foundation Trust, Preston, UK; ${ }^{3}$ Institute of Inflammation and Repair, Manchester Academic Health Sciences Centre, University of Manchester and National Institute of Health Research Respiratory and Allergy Clinical Research Facility, University Hospital of South Manchester, Manchester, UK

\subsection{6/thoraxjnl-2015-207770.430}

Background Patients that have chronic respiratory disease (CRD) and breathing pattern disorders (BPD) have a higher prevalence of anxiety and depression than the general population. These patients have worse respiratory health outcomes and in addition, their psychological problems are often left undiagnosed and untreated. Little is known about how anxiety and depression varies between CRD and BPD.

Methods This prospective study involved screening patients attending secondary and tertiary respiratory clinics over an eight-week period. Patients were asked to complete the Hospital Anxiety and Depression Scale (HADS), Short Form-12 (SF-12) and St. George's Respiratory Questionnaire (SGRQ). Demographical data and spirometry were also collected. Our primary outcome measure was the difference in these scores between patients with CRD (asthma, bronchiectasis and chronic obstructive pulmonary disease) compared to BPD (vocal cord dysfunction and dysfunctional breathing). 